\title{
A study protocol of a randomised controlled trial to measure the effects of an augmented prescribed exercise programme (APEP) for frail older medical patients in the acute setting
}

Ruth McCullagh ${ }^{1 *}$, Eimear O'Connell ${ }^{2}$, Sarah O'Meara ${ }^{3}$, Ivan Perry ${ }^{4}$, Anthony Fitzgerald ${ }^{4}$, Kieran $\mathrm{O}^{\prime} \mathrm{Connor}^{5}$, N. Frances Horgan ${ }^{6}$ and Suzanne Timmons ${ }^{1}$

\begin{abstract}
Background: Older adults experience functional decline in hospital leading to increased healthcare burden and morbidity. The benefits of augmented exercise in hospital remain uncertain. The aim of this trial is to measure the short and longer-term effects of augmented exercise for older medical in-patients on their physical performance, quality of life and health care utilisation.

Design \& Methods: Two hundred and twenty older medical patients will be blindly randomly allocated to the intervention or sham groups. Both groups will receive usual care (including routine physiotherapy care) augmented by two daily exercise sessions. The sham group will receive stretching and relaxation exercises while the intervention group will receive tailored strengthening and balance exercises. Differences between groups will be measured at baseline, discharge, and three months. The primary outcome measure will be length of stay. The secondary outcome measures will be healthcare utilisation, activity (accelerometry), physical performance (Short Physical Performance Battery), falls history in hospital and quality of life (EQ-5D-5 L).
\end{abstract}

Discussion: This simple intervention has the potential to transform the outcomes of the older patient in the acute setting.

Trial registration: ClinicalTrials.gov Identifier: NCT02463864, registered 26.05.2015.

Keywords: Frail, Medical, Inpatients, Exercise, Physiotherapy, Length of stay

\section{Background}

Older medical patients can experience a prolonged acute hospital stay and functional decline

In Ireland in 2011, $11.6 \%$ of the population was aged 65 years and over [1], and this is set to rise to $22 \%$ by 2041 [2]. Extended periods of poor health are predicted with this longevity [2]. Older patients occupy most acute hospital beds and most frequently experience a

\footnotetext{
* Correspondence: r.mccullagh@ucc.ie

${ }^{1}$ Centre for Gerontology \& Rehabilitation, University College Cork, Cork, Ireland

Full list of author information is available at the end of the article
}

prolonged length of stay (of greater than 30 days) [3], functional decline, high re-admission rates, falls, and institutionalisation [4]. Frailty is described as a geriatric syndrome with reduced capacity of the individual to resist stress and includes characteristics of slow mobility, low physical activity (PA) and energy levels [5]. Inactivity has been identified as a major determinant in the onset of frailty and exercise has been found to prevent or slow down this decline [6]. Therefore, maintenance of older adults' functional independence while in hospital is of utmost importance. 


\section{Physical activity levels and exercise intervention for medical patients in hospital}

Recent evidence has shown that older medical patients walk an average of $1534( \pm 112)$ steps per day in hospital and that prolonged length of stay was inversely associated with daily step count, even when adjusted for age, gender and physical performance on admission [7]. Similarly, Fisher et al. [8] found that older adults, who increase their walking activity by 600 steps on the second day of observation, were discharged home two days earlier. These findings suggest that low physical activity in hospital may directly influence length of stay and supports the theory that patients should exercise and remain active in the acute setting. Exercise programmes in hospital have been delivered independently or as a component of a multidisciplinary intervention and have been shown to improve physical performance, quality of life, reduce falls incidence and reduce healthcare utilisation [9-13].

\section{The evidence of effectiveness of augmented exercise in hospital}

To date, small benefits from augmented exercise on function and healthcare utilisation have been found. A systematic review found limited benefits from exercise as part of a multidisciplinary service on function, length of stay and discharge destination for acutely hospitalised older medical inpatients [14]. Three trials investigated the benefits of additional exercise alone [15-17]; none of which showed a significant improvement on length of stay. The authors suggested that the findings might have been weakened by using inappropriate outcome measures, recruitment of patients who had good baseline physical performance levels, and poor adherence to the exercise intervention that was being prescribed.

\section{Rationale for the trial and protocol}

To date, additional exercise has not been found to shorten frail older patients' hospital stay but the issues reported by previous authors may have weakened the results. To address these issues, the proposed protocol differs from previous studies in key parameters. A qualified physiotherapist will deliver and support the exercise sessions. Only patients who are less able to maintain physical activity will be recruited; those who need a walking aid and/or physical assistance on admission. Those who are unable to walk with assistance will be excluded from the trial. The Short Physical Performance Battery (SPPB) and walking speed will be used to measure physical performance, as these were previously found to be sensitive and appropriate for the study group [13]. The control arm will include sham exercises, to control for the considerable increase in patientphysiotherapist contact time. Finally, independent physical activity (usually walking) will be promoted outside the exercise sessions, in the intervention group.
Therefore, the aim of this study is to measure the effects of an augmented prescribed exercise programme for frail older medical inpatients on their physical performance, quality of life and healthcare utilisation.

\section{Methods \\ Design and study size}

The study is a single blind randomised controlled trial set in an acute 350-bedded teaching hospital. Power calculations based on the results of a pilot study indicated that a sample size of 200 (100 patients in each arm) would be required. To allow for an expected attrition rate of $5 \%$ [18], two hundred and twenty medical patients aged 65 years and over are randomly allocated to either the intervention or sham arm in a ratio of 1:1. (see Fig. 1). The study has been approved by the Clinical Research Ethics Committee of the Cork Teaching Hospitals. (ECM 3 (vv) 13/10/15).

\section{Selection of participants and allocation}

All suitable patients are screened and if eligible, are informed of the study and written consent is sought. The inclusion criteria are: medical patients aged 65 and over, who have been admitted from home and initially planned for discharge home, whose anticipated length of stay is greater than 3 days, and who require a mobility aid or assistance to walk. The exclusion criteria are: patients who have been an in-patient for more than $48 \mathrm{~h}$ prior to screening, who are unable to follow commands in the English language, unable to exercise with the assistance of one person only, bed or chair-bound at baseline, admitted with an acute psychiatric condition, require active end-of-life or critical care or when exercise is contraindicated.

To ensure adequate treatment time is given to each patient, recruitment is paused when there are five patients active in the trial. Based on the hospital's usual length of stay, this usually results in one patient recruited each weekday. If more than one patient is eligible for the study on one day, they are approached in chronological order of admission.

The patients are randomly allocated to either the intervention (APEP) or control group. A computer-generated random allocation sequence is used. Block randomisation is applied (in groups of approximately 50 patients). Post hoc power analysis will be calculated when the first seventy-five patients have completed the trial.

\section{Roles of the researchers}

Randomisation and data entry is completed by the Research Assistant (RA, SO'M). Screening, recruitment, baseline measurements and all exercise sessions are completed by the Principal Investigator (PI, $\mathrm{RMcC}$ ). The discharge and follow-up assessments are completed by the blinded Research Physiotherapist (RPT, EO'C), who has no involvement in either the allocation or intervention components of the trial. 


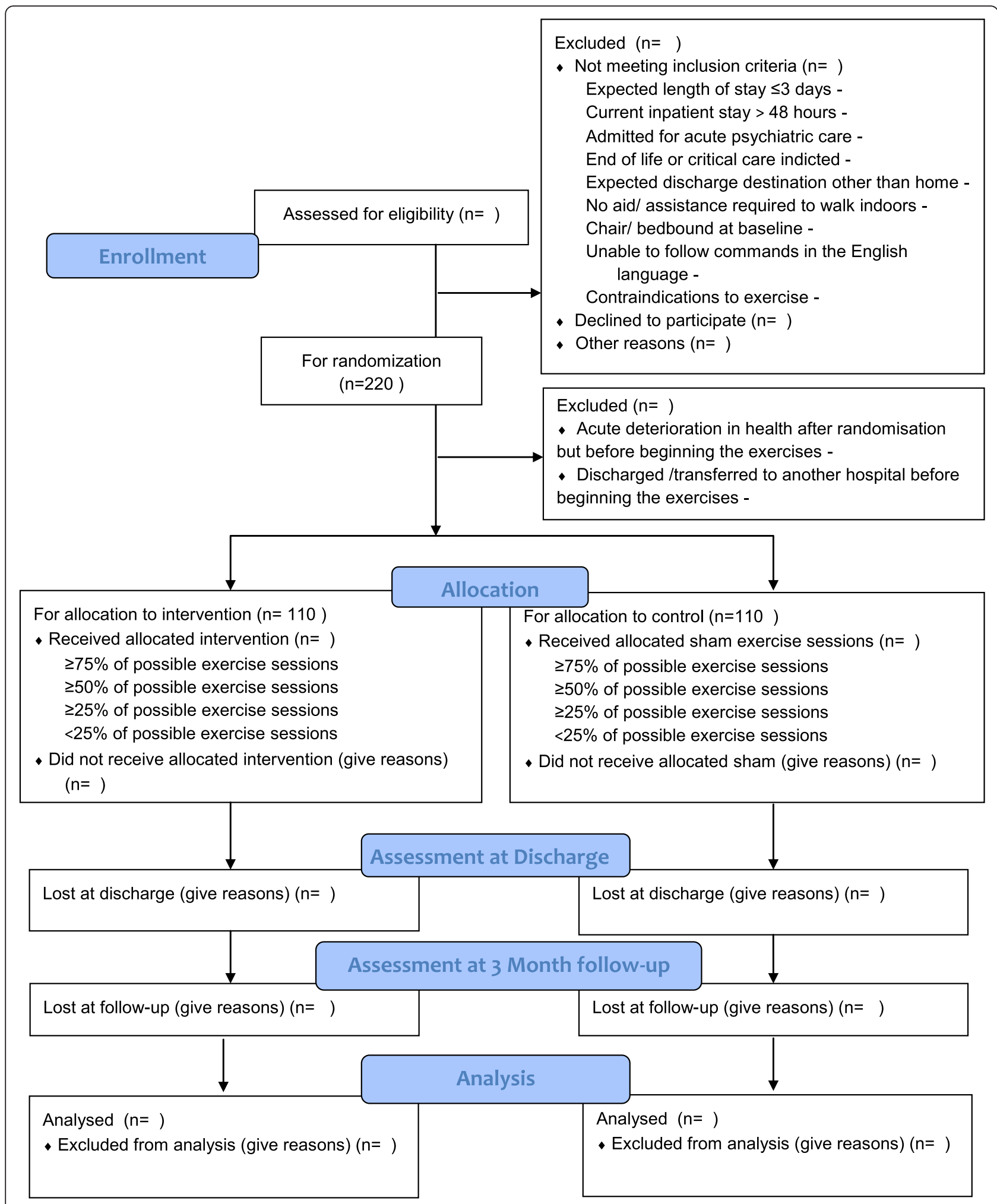

Fig. 1 Flowchart with details of the study design and flow of participants

\section{Measurements}

Patients are assessed within $48 \mathrm{~h}$ of admission, at discharge and at three months following discharge. The assessment tools are described in Table 1. Baseline data includes demographics, co-morbidity, medication use and home circumstances. 
Table 1 Summary of the Measurements used in the Study

\begin{tabular}{lll}
\hline Domain & On admission & Discharge and 3 months \\
\hline Medical Morbidity & Cumulative Illness Rating Scale (CIRS-G [19]); & \\
& Total number of medications & Grip Strength (kgs) \\
Frailty & SHARE- FI [20] & SPPB [22] (includes walking speed) \\
Physical Performance & Short Physical Performance Battery & \\
& (SPPB) (includes walking speed) [22] & Number of falls and injuries sustained \\
Falls Efficacy and Self-Reported Functional Ability & Number of Falls and injuries sustained & Nottingham Extended Activities of Daily \\
& Falls Efficacy Scale - International (FES-I) [25] & Living Scale (N-EADL) [26] \\
& & \\
& Nottingham Extended Activities of Daily & \\
Cognition & Living Scale (N-EADL) [26] & 6CIT [21] \\
Quality of Life & 6CIT [21] & EQ-5D-5 L [24] \\
Physical Activity & EQ-5D-5 L [24] & Accelerometers (Stepwatch Activity Monitor, \\
& SAM) during hospitalisation only &
\end{tabular}

The primary outcome measure is length of stay, a key healthcare utilisation metric. The secondary outcome measures includes patient-related measures: changes in physical performance (SPPB, walking speed), and quality of life at 3 months following discharge, differences in physical activity levels between groups in hospital (based on accelerometry data) and re-admission rates over three months. The baseline assessment is designed to capture frailty, co-morbidity and disability. Measurements that are appropriate and quick to administer have been chosen to limit patient fatigue.

\section{Measurements of Co-morbidity and frailty}

The Cumulative Illness Rating Scale Geriatrics (CIRS-G) has been chosen as it measures chronic medical illness burden with good reliability and is a validated tool both as an indicator of health status and as a predictor of 18month mortality and hospitalisation [19]. The CIRS-G has 14 categories, with a 0 to 4 grading system of impairment in each organ system. The score may theoretically range from 0 to 56 , with a higher score reflecting greater impairment in several systems.

To measure frailty, the SHARE-FI [20] tool has been chosen as it is a valid and simple measurement of frailty. Five SHARE variables approximating Fried's frailty definition are used: fatigue, loss of appetite, grip strength, functional difficulties and physical activity. Scores range between 2.7 to 13.4 and the SHARE-FI calculators (genderspecific) are freely available on the web to interpret the level of frailty [20].

The Six-Item Cognitive Impairment Test (6-CIT) will be used to measure cognition as it is quick to administer, its diagnostic accuracy is as high as the Mini-Mental State Examination when used in the acute hospital setting, and it is not sensitive to an educational level and does require advanced language skills [21].

\section{Measurements of disability}

Physical Performance is measured using the Short Physical Performance Battery (SPPB), which includes walking speed [22]. The SPPB has been chosen as it is quick, practical and safe to use with this population. The scores range from 0 (unable to stand independently,) to 12 (independent tandem balance for $10 \mathrm{~s}$, able to walk $4 \mathrm{~m}$ within $4.82 \mathrm{~s}$ and sit to stand 5 times in $11 \mathrm{~s}$ ). Walking speed is known to be a strong indicator of patients' physical performance and is an independent predictor of survival and institutionalisation [23]. All patients scoring less than 1 on the SPPB will be eliminated from the study to allow us to detect functional decline while in hospital.

\section{Measurements of well-being, self-efficacy and self- reported functional ability}

Quality of Life will be measured using the EuroQol 5Domain 5-Level Scale (EQ-5D-5 L) [24] as it is wellused and easy to administer. The five domains assessed are mobility, self-care, usual activities, pain/discomfort, anxiety/depression, and a visual analogue scale, ranging from 0 to 100, to measure self-rated health status [24].

At baseline only, fear of falling is measured using the Falls Efficacy Scale-International (FES-I) [25]. This tool consists of 14 activity-related questions. The questions aim to determine how concerned older adults are about falling while performing these activities on a scale of 0 (not concerned at all) to 4 (very concerned).

The Nottingham Extended Activities of Daily Living Scale (N-EADL) [26] is a self-reported tool to measure the patients' ability to complete 16 community-based activities. On admission, patients are asked to report their functional ability both pre-morbidly (before the onset of the illness) and on admission (the day before they were admitted) and again, at the three month follow-up assessment. Patients can score 0 (unable to complete the 
activity with/without help) or 1 (able to complete the score with/without help). This has been used extensively in older adult populations, including patients with stroke and fallers $[27,28]$.

\section{Measurements of physical activity}

All patients with good skin condition at the ankle are asked to wear accelerometers (Stepwatch Activity Monitors, SAM) to measure physical activity (step-count) in the hospital. These devices have been validated in frail older in-patients and can be worn in the shower, helping compliance and successful data collection [29]. They are capable of storing up to seven days of data without interruption using a $15 \mathrm{~s}$ epoch. They are attached on the first day of recruitment and worn continuously while in hospital or for the first seven days. All staff are informed of their application. The accelerometry data will be analysed to measure

1. Changes in activity between groups.

2. Levels of physical activity compared to a recently completed observation study (which assisted in identifying those who were at risk/not at risk of functional decline).

\section{Procedure}

\section{Intervention and routine care schedule}

Both groups receive usual multidisciplinary care. The medical team refer patients to physiotherapy if required. It is delivered, an average of three times weekly, by the clinical ward physiotherapist and will be routine in nature. It consists of assessment, discharge planning, exercise, provision of aids and rehabilitation. Both the control and the intervention groups also receive two augmented, twenty minute to half-hour exercise sessions (tailored to the patient's endurance), five days per week, delivered on a one-to-one basis by the PI.

\section{Consent, assessment and exercise procedure}

Upon screening, the medical team are contacted to confirm that there is no medical contra-indication to exercise for the patient. Eligible patients are informed of the study verbally and given a copy of the patient information leaflet. They give written informed consent to the study, including access to their medical notes, assessment at baseline, outcome and follow-up, and the twice daily exercise sessions. If the patient is considered to be cognitively impaired by the medical or nursing staff, the patients' written consent is augmented by verbal assent from their next of kin. Patients with severe confusion, who are unable to follow commands, or are agitated, are not recruited to the study.

If recruited, patients are assessed (Time 1), and through concealed allocation, randomly allocated to the control group or the intervention group at that time. All recruited patients, who consent to wearing the accelerometer and with good skin condition at the ankle, are fitted with the SAM to measure physical activity in hospital.

For those in the intervention (Augmented Prescribed Exercise Programme, APEP) group, their exercise programme is prescribed to address their physical limitations identified through the assessment. The exercises are chosen to improve strength and balance, core stability, sit-to-stand function, balance (in standing and walking), walking and endurance. The intervention group are also actively encouraged to mobilise while in hospital, with assistance when necessary, and provided with walking aids initially, if required. The sham exercise sessions for the control group are not prescribed but consist of standardised stretching and relaxation exercises.

All exercise sessions begin within $24 \mathrm{~h}$ of group allocation and continue until the day before discharge. Verbal consent for each session is sought.

To ensure false step-count does not occur, the accelerometer (SAM) is turned upside-down (in this position, it is unable to record steps) when the patient is exercising at the bedside, i.e., not walking, and returned to the upright position before walking or at the end of the session.

Patient compliance, exercise prescription and session duration is recorded. Within one day of planned discharge, all patients are re-assessed and the accelerometer (SAM) is removed from the patient by the RPT (Time 2; see Table 1). Patients who are deemed for long-term care (as they are unable to manage at home) or for end-oflife care are re-assessed on the date that the decision is made and those results are used.

The patients are reassessed at three months post discharge, by the RPT (Time 3, see Table 1). New onset of illness, physical performance, walking speed, quality of life and self-reported functional ability is measured. Hospital and Accident and Emergency utilisation since discharge is recorded.

\section{Safety, reporting of adverse events and serious adverse events}

The main adverse events anticipated in this study are skin rashes from the accelerometer, and falls, cardiac ischaemia or pulmonary embolism during exercise. All adverse events are recorded using an adverse event recording worksheet, and causality to the study intervention is determined, in consultation with the treating physician, by a study physician $(\mathrm{KO} C)$. The Sponsor's Clinical Research Supporting Officer is notified electronically, within $24 \mathrm{~h}$, of any serious adverse event that occurs during the trial. From a previous local longitudinal study, the Cork Dementia Study, the in-hospital mortality of this cohort is expected to be approximately two per cent [18]. This predicts approximately 5 deaths of trial subjects. However, the type of exercise involved is similar to usual care, 
patients with a contraindication to exercise will be excluded at source, and patients who are unwell on a particular day will not exercise.

\section{Statistical analysis}

The results will be analysed and presented as recommended by the CONSORT guidelines [30]. The primary outcome measure will be length of stay. This will be described using Kaplan-Meier "survival" curves and the results between groups will be compared using a log-rank test. Univariate and multivariate linear regression analysis will be used to determine differences in physical activity in hospital and physical performance, quality of life at discharge and three months post-discharge, and readmission rates at three months.

This analysis will help to define whether a simple physiotherapy-led exercise intervention will shorten length of stay, increase physical activity in hospital, limit functional decline and readmission rates and improve quality of life in frail older hospitalised patients.

\section{Discussion}

This study has been designed to measure the effects of an augmented prescribed exercise programme for frail older hospitalised patients. The study design is based upon results of an earlier pilot study and issues reported from previously published studies. Therefore, this protocol differs from previous studies in three key areas: patient selection, intervention and outcome measurements.

Previous studies included some patients who were fully independently mobile but de Morton et al. [31] found that the intervention was most effective for those requiring an aid or assistance to walk. For this reason, we will exclude those who are independently mobile. For pragmatic reasons, we will also exclude those unable to walk at baseline, i.e., bed or chair bound.

There is strong evidence of low physical activity in hospital [32, 33] and Broderick et al. [34] found that many of the barriers could be addressed easily. For these reasons, we will not only deliver supervised exercise sessions, but in addition, will encourage mobility while in hospital and provide walking aids initially, if required. The exercises are designed to improve physical performance, transfer function, walking, balance and strength in order to maintain functional mobility as much as possible.

Our pilot study [13] showed that there was a considerable difference in physiotherapy contact time with the intervention, possibly introducing a Hawthorne effect. This has been addressed by using a sham intervention for the control group. To the authors' knowledge, this is the first time that a sham intervention has been included in this type of study.

The intervention will be delivered by a senior physiotherapist. The interventions of previous studies have been delivered by a physiotherapist assistant or a physiotherapy student, under the guidance of a qualified physiotherapist $[13,16,17]$. However, patients' physical performance fluctuates in the acute setting. If a qualified physiotherapist delivers the programme, it allows the optimal intervention to be delivered on a daily basis. It will also allow a greater opportunity to advise the patient regarding their independent physical activity in hospital and to address barriers to the intervention delivery such as pain relief.

Up to $27 \%$ of the patients were unable to complete the Timed Up and Go Test in previous studies $[16,17]$. Therefore, the Short Physical Performance Battery will be used, which was found to be feasible and sensitive to change in a previous pilot study [13]. Length of stay will be used as the primary outcome measure as this is available electronically and absolutely complete data. A high attrition rate is predicted for the three-month follow-up attendance as our study group consist of frailer older adults.

A small number of studies have shown that interventions to increase older medical inpatients' physical activity can be modestly beneficial. Previous authors discuss issues such as patient selection, intervention type and outcome measures. This protocol has been designed to include the frailer patient, to include a tailored and comprehensive intervention, and to measure the effects with the most valid outcome measure.

\section{Abbreviations}

6-CIT: Six-Item Cognitive Impairment Test; APEP: Augmented prescribed exercise programme; CIRS-G: Cumulative Illness Rating Scale - Geriatrics; EQ-5D-5 L: EuroQol 5-Domain 5-Level Scale; FES-I: Falls Efficacy Scale International; N-EADL: Nottingham Extended Activities of Daily Living Scale; Pl: Principal Investigator;

RA: Research Assistant; RPT: Research Physiotherapist; SAM: Stepwatch Activity Monitor; SHARE-FI: Survey of Health, Aging and Retirement in Europe Frailty Instrument; SPPB: Short Physical Performance Battery.

\section{Competing interests}

The authors declare that there are no conflicts of interest. This study has not received funding or assistance from a commercial organisation.

\section{Authors' contributions}

RMcC: concept and design, acquisition of subjects and baseline data collection, delivery of the intervention, analysis and interpretation of the data, manuscript preparation. EO'C: outcome data acquisition, manuscript preparation. SO'M patient allocation process, data entry, manuscript preparation. IP: concept and design, manuscript preparation. AF: concept and design, data analysis. KO'C: concept and design, safety monitoring, manuscript preparation. NFH: concept and design, study oversight, manuscript preparation. ST: concept and design, study oversight, analysis and interpretation of the data, manuscript preparation. All authors read and approved the final manuscript.

\section{Authors' details}

RMcC, Senior Physiotherapist and PhD Student, Centre for Gerontology \& Rehabilitation, University College Cork, Ireland. EO'C, Physiotherapist, Physiotherapy Department, Mercy University Hospital, Cork, Ireland. SO'M, Research Assistant, Clinical Research Facility, Mercy University Hospital, Cork, Ireland. IP, Professor of the Dept. of Epidemiology \& Public Health, University College Cork, Ireland. AF, Senior Statistician, Dept. of Epidemiology \& Public Health, University College Cork, Ireland. KO'C, Consultant Geriatrician, Mercy University Hospital, Cork, Ireland. NFH, Senior Lecturer, School of Physiotherapy, Royal College of Surgeons in Ireland, Dublin, Ireland. ST, Consultant Geriatrician, Mercy University Hospital and Senior Lecturer, Centre for Gerontology \& Rehabilitation, University College Cork, Ireland, Ireland. 


\section{Acknowledgements}

This study is funded by the Health Research Board of Ireland, following a peerreviewed competition, as a Research Fellowship Training Grant (HPF 2013 451). We would like to thank the Clinical Research Facility and the ward staff at the Mercy University Hospital for facilitating this study.

\section{Author details ${ }^{3}$ Clinical Research Facility, Mercy University Hospital, Cork, Ireland. ${ }^{4}$ Epidemiology \& Public Health, University College Cork, Cork, Ireland. Ireland. \\ Received: 24 November 2015 Accepted: 2 April 2016 \\ Published online: 08 April 2016}

${ }^{1}$ Centre for Gerontology \& Rehabilitation, University College Cork, Cork, Ireland. ${ }^{2}$ Physiotherapy Department, Mercy University Hospital, Cork, Ireland. ${ }^{5}$ Department of Geriatric Medicine, Mercy University Hospital, Cork, Ireland. 'School of Physiotherapy, Royal College of Surgeons in Ireland, Dublin,

\section{References}

1. CSO: Census 2011 Profile 2 - Older and Younger. In: Central Statistics Office Census 2011 Reports. http://www.cso.ie/en/census/census2011 reports/ census2011 profile2-olderandyounger/. Accessed 6 March 2016.

2. McGill P: Illustrating Ageing in Ireland North and South: Key Facts and Figures Belfast: Centre for Ageing Research and Development in Ireland. 2010

3. ESRI: Activity in Acute Public Hospitals in Ireland, Annual Report, 2012. https://www.esri.ie/news/activity-in-acute-public-hospitals-in-ireland-2012annual-report/. Accessed 6 Mar 2016

4. Covinsky KE, Palmer RM, Fortinsky RH, Counsell SR, Stewart AL, Kresevic D, Burant CJ, Landefeld CS. Loss of independence in activities of daily living in older adults hospitalized with medical illnesses: increased vulnerability with age. J Am Geriatr Soc. 2003;51(4):451-8.

5. Fried LP, Tangen CM, Walston J, Newman AB, Hirsch C, Gottdiener J, Seeman T, Tracy R, Kop WJ, Burke G et al. Frailty in older adults: evidence for a phenotype. J Gerontol A Biol Sci Med Sci. 2001;56:M146-156.

6. Boyd CM, Xue QL, Simpson CF, Guralnik JM, Fried LP. Frailty, hospitalization, and progression of disability in a cohort of disabled older women. Am J Med. 2005;118:1225-31.

7. McCullagh R, Dillon C, Horgan NF, Timmons S: Determinants of Prolonged Lenth of Stay and Functional Decline of Older Hospitalised Patients. In: 4th International Conference on Ambulatory Monitoring of Physical Activity and Movement (ICAMPAM): 2015; Limerick. 44

8. Fisher SR, Kuo YF, Graham JE, Ottenbacher KJ, Ostir GV. Early ambulation and length of stay in older adults hospitalized for acute illness. Arch Intern Med. 2010;170(21):1942-3.

9. Jones CT, Lowe AJ, MacGregor L, Brand CA. A randomised controlled trial of an exercise intervention to reduce functional decline and health service utilisation in the hospitalised elderly. Australas J Ageing. 2006;25(3):126-33.

10. Slaets JP, Kauffmann RH, Duivenvoorden HJ, Pelemans W, Schudel WJ. A randomized trial of geriatric liaison intervention in elderly medical inpatients. Psychosom Med. 1997:59(6):585-91.

11. Counsell SR, Holder CM, Liebenauer LL, Palmer RM, Fortinsky RH, Kresevic DM, Quinn LM, Allen KR, Covinsky KE, Landefeld CS. Effects of a multicomponent intervention on functional outcomes and process of care in hospitalized older patients: a randomized controlled trial of Acute Care for Elders (ACE) in a community hospital. J Am Geriatr Soc. 2000;48(12):1572-81.

12. Haines TP, Bennell KL, Osborne RH, Hill KD. Effectiveness of targeted falls prevention programme in subacute hospital setting: randomised controlled trial. BMJ. 2004;328(7441):676

13. McCullagh R, Fitzgerald E, O'Connor K, Broderick L, Kennedy C, O'Reilly N, Martin R, Timmons S. The functional decline of hospitalised older patients are we doing enough? Physiother Pract Res. 2014;35:141-2.

14. de Morton NA, Keating JL, Jeffs $K$. The effect of exercise on outcomes for older acute medical inpatients compared with control or alternative treatments: a systematic review of randomized controlled trials. Clin Rehabil. 2007;21(1):3-16.

15. Siebens $\mathrm{H}$, Aronow $\mathrm{H}$, Edwards D, Ghasemi Z. A randomized controlled trial of exercise to improve outcomes of acute hospitalization in older adults. J Am Geriatr Soc. 2000;48:1545-52.

16. de Morton NA, Keating JL, Berlowitz DJ, Jackson B, Lim WK. Additional exercise does not change hospital or patient outcomes in older medical patients: a controlled clinical trial. Aust J Physiother. 2007;53:105-11.
17. Mudge AM, Giebel AJ, Cutler AJ. Exercising body and mind: an integrated approach to functional independence in hospitalized older people. J Am Geriatr Soc. 2008;56(4):630-5.

18. Manning E, Timmons S, Barrett A, Browne V, Molloy DW, O'Regan N, Cahill S, Linehan J, O'Sullivan K, Woods N: The Influence of Dementia on One-Year Mortality Following Hospital Admission, and Place and Cause of Death. In: Irish Gerontogical Society Conference: 2014. Ir J Med Sci S319-S320.

19. Salvi F, Miller MD, Grilli A, Giorgi R, Towers AL, Morichi V, Spazzafumo L, Mancinelli $L$, Espinosa $E$, Rappelli A et al. A manual of guidelines to score the modified cumulative illness rating scale and its validation in acute hospitalized elderly patients. J Am Geriatr Soc. 2008;56(10):1926-31.

20. Romero-Ortuno R, Walsh CD, Lawlor BA, Kenny RA. A frailty instrument for primary care: findings from the Survey of Health, Ageing and Retirement in Europe (SHARE). BMC Geriatr. 2010;10:57.

21. Tuijl JP, Scholte EM, de Craen AJ, van der Mast RC. Screening for cognitive impairment in older general hospital patients: comparison of the Six-item cognitive impairment test with the mini-mental state examination. Int J Geriatr Psychiatry. 2012;27(7):755-62.

22. Guralnik JM, Simonsick EM, Ferrucci L, Glynn RJ, Berkman LF, Blazer DG, Scherr PA, Wallace RB. A short physical performance battery assessing lower extremity function: association with self-reported disability and prediction of mortality and nursing home admission. J Gerontol. 1994:49:M85-94.

23. Ostir GV, Berges IM, Kuo YF, Goodwin JS, Fisher SR, Guralnik JM. Mobility activity and its value as a prognostic indicator of survival in hospitalized older adults. J Am Geriatr Soc. 2013;61:551-7.

24. van Hout B, Janssen MF, Feng YS, Kohlmann T, Busschbach J, Golicki D, Lloyd A, Scalone L, Kind P, Pickard AS. Interim scoring for the EQ-5D-5 L: mapping the EQ-5D-5 L to EQ-5D-3 L value sets. Value Health. 2012;15(5):708-15.

25. Yardley L, Beyer N, Hauer K, Kempen G, Piot-Ziegler C, Todd C. Development and initial validation of the Falls Efficacy Scale-International (FES-I). Age Ageing. 2005:34:614-9.

26. Lincoln NB, Gladman JRF. The extended activities of daily living scale: a further validation. Disabil Rehabil. 1992;14(1):41-3.

27. Fairhall N, Aggar C, Kurrle S, Sherrington C, Lord S, Lockwood K, Monaghan N, Cameron I. Frailty Intervention Trial (FIT). BMC Geriatr. 2008;8(1):27.

28. Galvin R, Cusack T, Stokes E. A randomised controlled trial evaluating family mediated exercise (FAME) therapy following stroke. BMC Neurol. 2008;8:22

29. McCullagh R, O'Connell AM, Dillon C, Horgan F, Timmons S: Comparative Accuracy of Motion Sensors for Frail-Older Hospitalised Patients. In: The International Association of Gerontology and Geriatrics European Region Congress 2015, Dublin, Ireland. 1325

30. Moher D, Hopewell S, Schulz KF, Montori V, Gøtzsche PC, Devereaux PJ, Elbourne D, Egger M, Altman DG. CONSORT 2010 explanation and elaboration: updated guidelines for reporting parallel group randomised trials. J Clin Epidemiol. 2010;63(8):e1-37.

31. De Morton NA, Jones CT, Keating JL, Berlowitz DJ, MacGregor L, Lim WK, Jackson B, Brand CA. The effect of exercise on outcomes for hospitalised older acute medical patients: an individual patient data meta-analysis. Age and ageing. 2007;36(2):219-22.

32. Brown CJ, Redden DT, Flood KL, Allman RM. The underrecognized epidemic of low mobility during hospitalization of older adults. J Am Geriatr Soc. 2009:57:1660-5.

33. Fisher SR, Goodwin JS, Protas EJ, Kuo YF, Graham JE, Ottenbacher KJ, Ostir GV. Ambulatory activity of older adults hospitalized with acute medical r illness. J Am Geriatr Soc. 2010;59:91-5.

34. Broderick L, Savage E, McCullagh R, Bantry-White E, Timmons S: Influences on Exercise Behaviours of Frail Older Adults in the Acute Setting. In: Proceedings from the Irish Geronotological Society Conference: 2013; Dublin. Ir J Med Sc 2013 182: S225-S225. 